\title{
Surface Activity-Tuned Metal Oxide Chemiresistor: Toward Direct and Quantitative Halitosis Diagnosis
}

Hamin Shin, ${ }^{\dagger, 1,2}$ Dong-Ha Kim, ${ }^{\dagger, 1,2}$ Wonjong Jung, ${ }^{\dagger, 4}$ Ji-Soo Jang, ${ }^{1,2,3}$ Yoon Hwa Kim, ${ }^{1,2}$ Yeolho Lee, ${ }^{4}$ Kiyoung Chang, ${ }^{4}$ Joonhyung Lee, ${ }^{4}$ Jongae Park, ${ }^{4}$ Kak Namkoong, ${ }^{4, *}$ and Il-Doo Kim ${ }^{1,2, *}$

Prof. I.-D. Kim, H. Shin, D.-H. Kim, Dr. J.-S. Jang, Dr. Y. H. Kim

${ }^{1}$ Department of Materials Science and Engineering, Korea Advanced Institute of Science and Technology (KAIST), 291 Daehak-ro, Yuseong-gu, Daejeon 34141, Republic of Korea

${ }^{2}$ Membrane Innovation Center for Anti-Virus \& Air-Quality Control, KI Nanocentury, KAIST, 291, Daehak-ro, Yuseong-gu, Daejeon 34141, Republic of Korea

${ }^{3}$ Department of Chemical and Environmental Engineering, Yale University, New Haven, Connecticut 06520-8286, United States

Dr. K. Namkoong, W. Jung, Y. Lee, K. Chang, J. Lee, J. Park

${ }^{4}$ Healthcare Sensor Lab., Device Research Center, Samsung Advanced Institute of Technology, Samsung Electronics Co., Ltd., 130 Samsung-ro, Yeongtong-gu, Suwon-si, Gyeonggi-do 16678, Republic of Korea

*Corresponding author e-mail: kak.namkoong@samsung.com and idkim@kaist.ac.kr

These authors contributed equally to this work.

Keywords: sodium; metal oxide nanofiber; gas sensor; hydrogen sulfide; direct breath analysis 


\section{Table of Contents}

Figure S1. TEM images of Pt NPs.

Figure S2. TG and DSC analysis of as-spun composite NFs.

Figure S3. SEM images of $\mathrm{NaPt}-\mathrm{WO}_{3} \mathrm{NFs}$.

Figure S4. Labelled SAED pattern

Figure S5. TEM images of pristine $\mathrm{WO}_{3} \mathrm{NFs}$.

Figure S6. Temperature-dependent sensing performance of $\mathrm{NaPt}-\mathrm{WO}_{3} \mathrm{NFs}$.

Figure S7. $\mathrm{H}_{2} \mathrm{~S}$ sensing performance of $\mathrm{Na} 1-\mathrm{WO}_{3} \mathrm{NFs}$.

Figure S8. Humidity effect on $\mathrm{NaPt}-\mathrm{WO}_{3} \mathrm{NFs}$.

Figure S9. Response time graph of $\mathrm{NaPt}-\mathrm{WO}_{3} \mathrm{NFs}$.

Figure S10. Selectivity graph of $\mathrm{NaPt}-\mathrm{WO}_{3} \mathrm{NFs}$.

Figure S11. Long-term reliability tests of $\mathrm{NaPt}-\mathrm{WO}_{3} \mathrm{NFs}$.

Figure S12. Extended long-term reliability test (116 cycles).

Figure S13. XPS analysis of $\mathrm{NaPt}-\mathrm{WO}_{3} \mathrm{NFs}$ in the vicinity of $\mathrm{S} 2 p$ after recovery.

Figure S14. XPS analysis of $\mathrm{NaPt}-\mathrm{WO}_{3} \mathrm{NFs}$ in the vicinity of Pt4 $f$ peaks.

Figure S15. UPS analysis of $\mathrm{NaPt}-\mathrm{WO}_{3} \mathrm{NFs}$ and pristine $\mathrm{WO}_{3} \mathrm{NFs}$.

Figure S16. Hardware block diagram of the overall system with the test device (gray box) and PCB artwork of the test device.

Figure S17. Detailed procedure for oral $\mathrm{H}_{2} \mathrm{~S}$ concentration measurement using OralChroma.

Figure S18. Correlation studies with $\mathrm{CH}_{3} \mathrm{SH}$ and $\mathrm{C}_{2} \mathrm{H}_{6} \mathrm{~S}$ using the same fitting model as $\mathrm{H}_{2} \mathrm{~S}$.

Figure S19. Schematic illustration of the gas sensor measurement setup.

Table S1. ICP results of relative amounts (wt\%) of $\mathrm{Na}$ and $\mathrm{Pt}$ in the prepared samples with respect to $\mathrm{WO}_{3}$.

Table S2. Raw $\mathrm{H}_{2} \mathrm{~S}$ concentration data obtained by direct, real-time human breath experiments using OralChroma and the developed test device.

Table S3. Accuracy of the developed test device compared with OralChroma. 


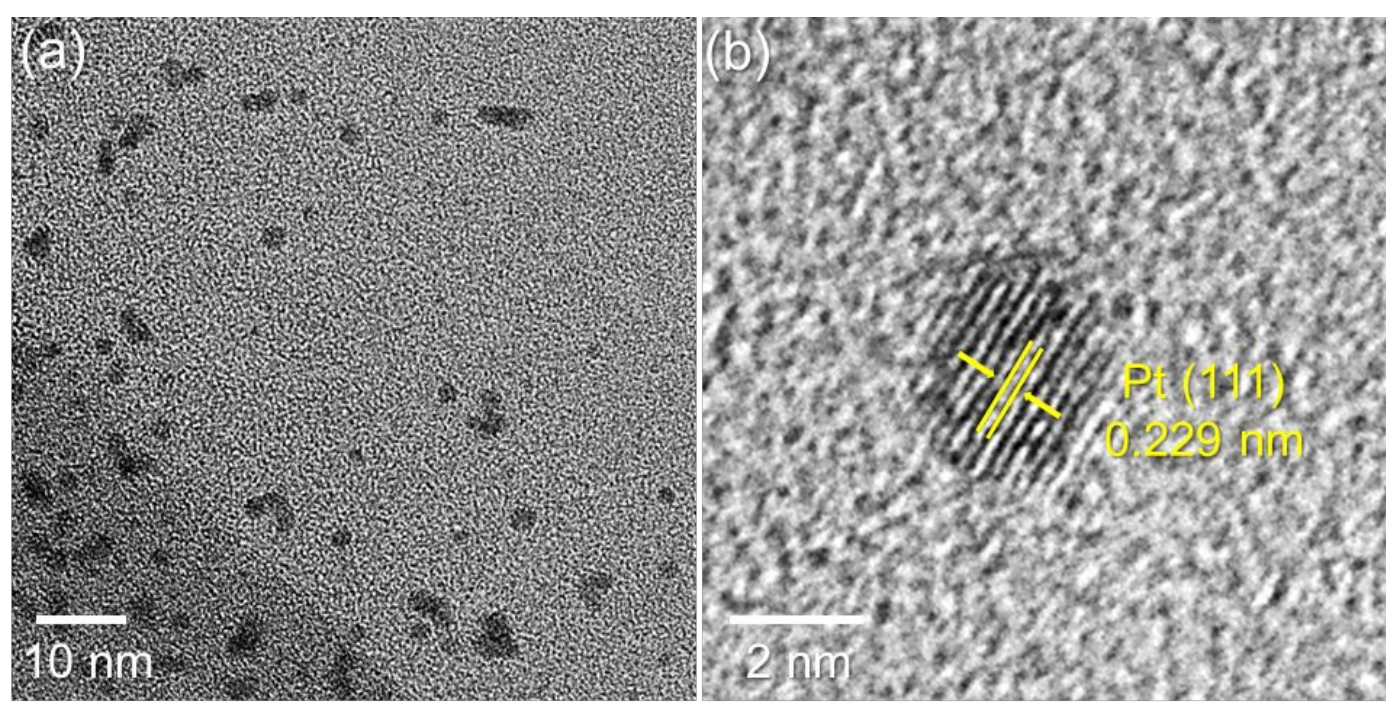

Figure S1. TEM images of Pt NPs encapsulated in a protein template. 

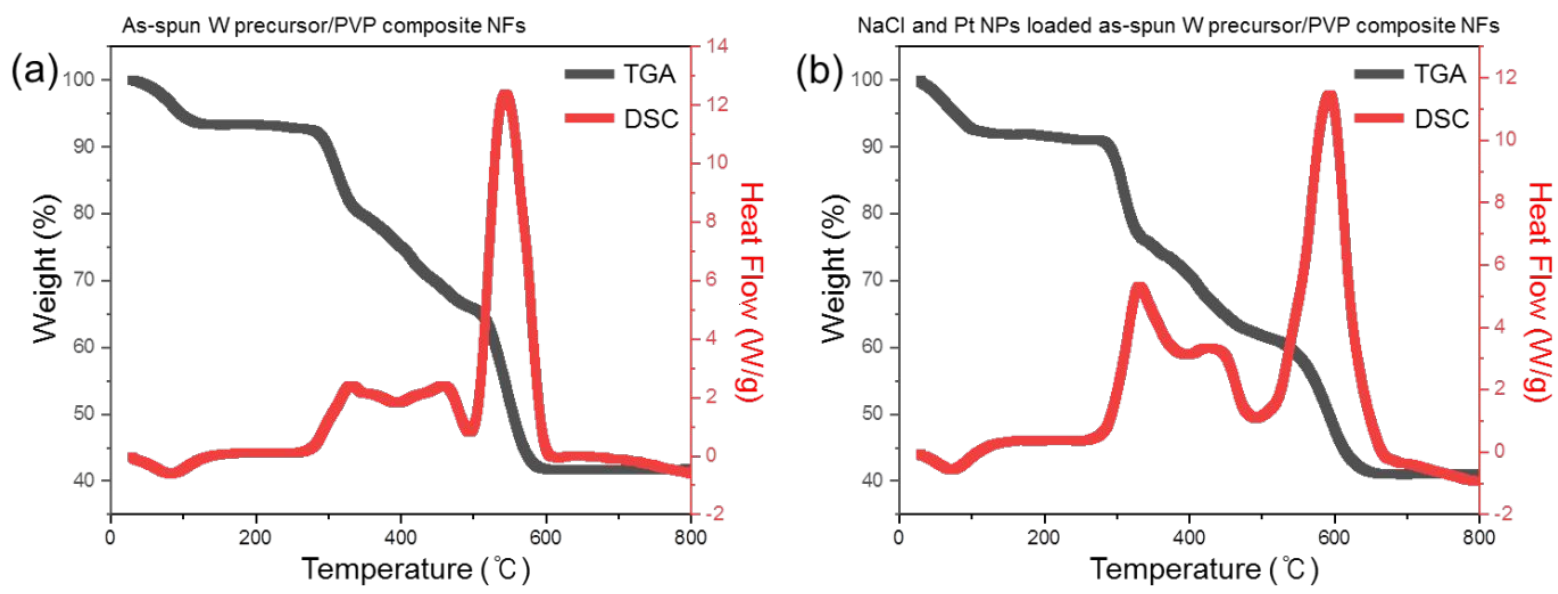

Figure S2. TG and DSC analysis of the (a) as-spun W precursor/PVP composite NFs and (b) as-spun $\mathrm{W}$ precursor/PVP composite NFs loaded with $\mathrm{NaCl}$ and Pt NPs in the temperature range of $30-600{ }^{\circ} \mathrm{C}$. 


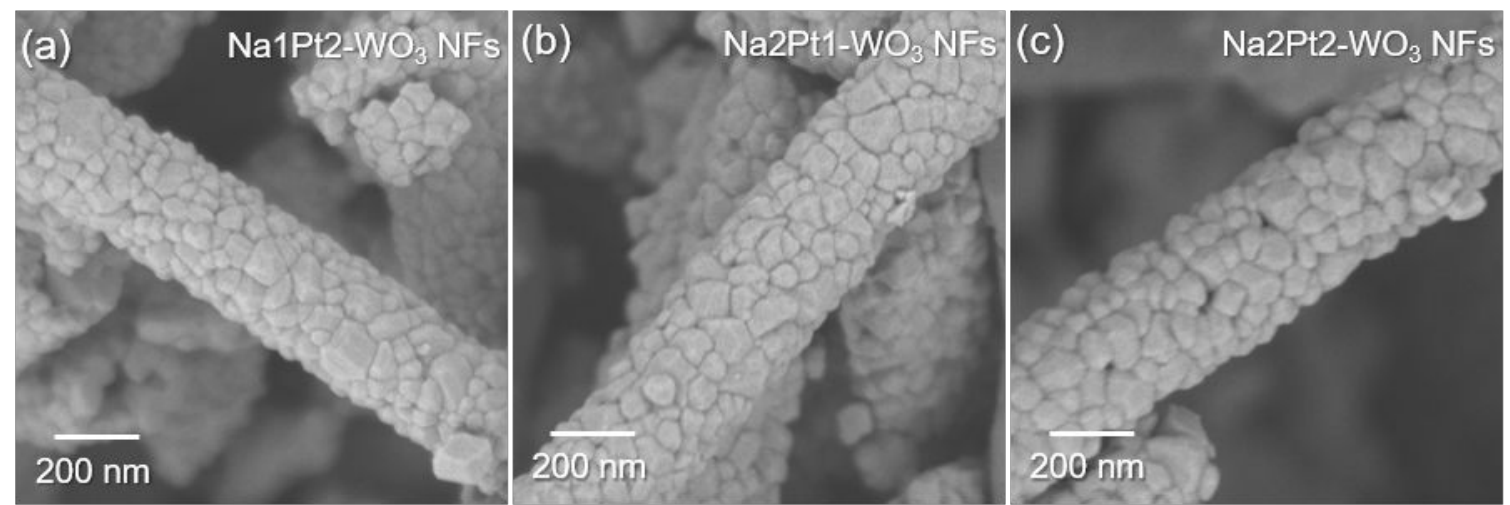

Figure S3. SEM images of (a) Na1Pt2- $\mathrm{WO}_{3} \mathrm{NFs}$, (b) Na2Pt1- $\mathrm{WO}_{3} \mathrm{NFs}$, and (c) Na2Pt2- $\mathrm{WO}_{3}$ NFs. 


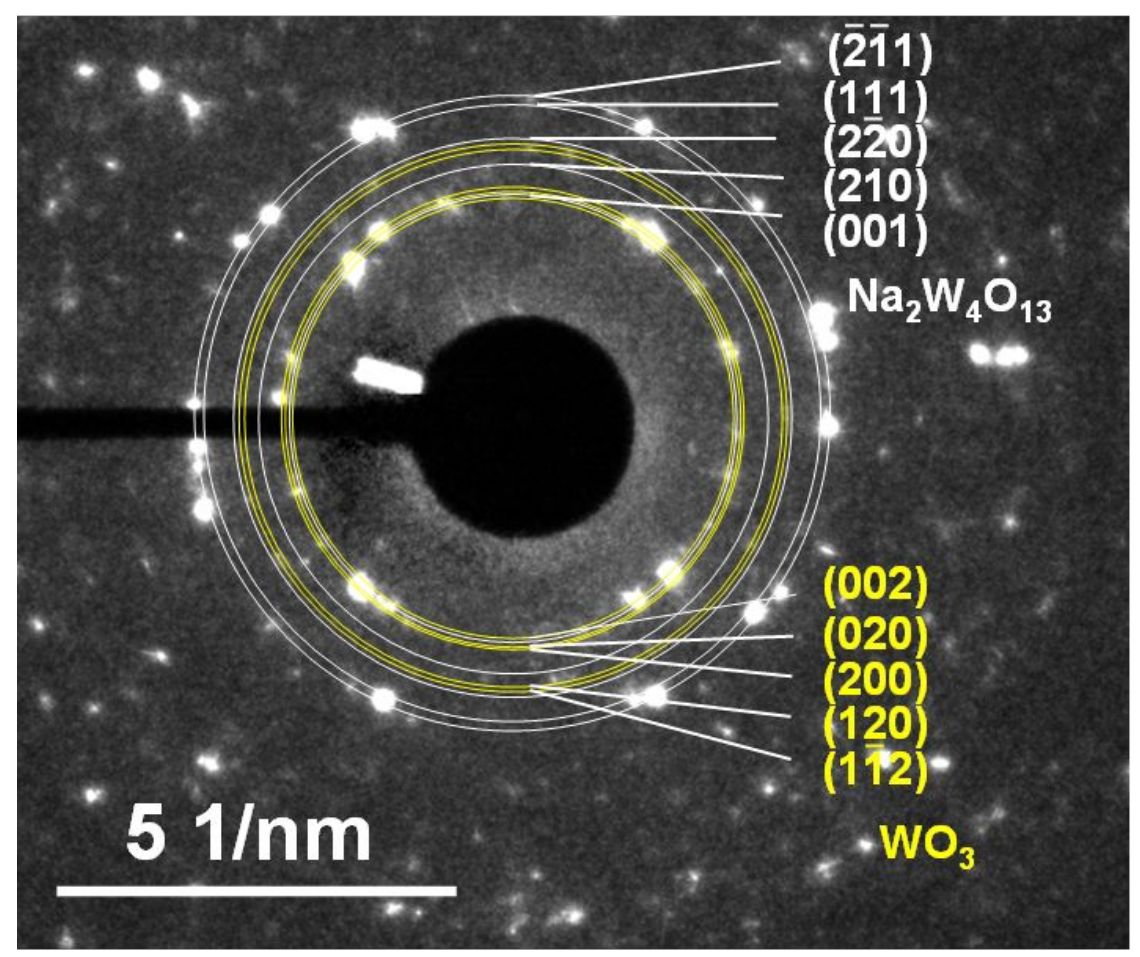

Figure S4. Selected-area electron diffraction (SAED) pattern labelled with the crystalline $\mathrm{Na}_{2} \mathrm{~W}_{4} \mathrm{O}_{13}$ and $\mathrm{WO}_{3}$ structures. 


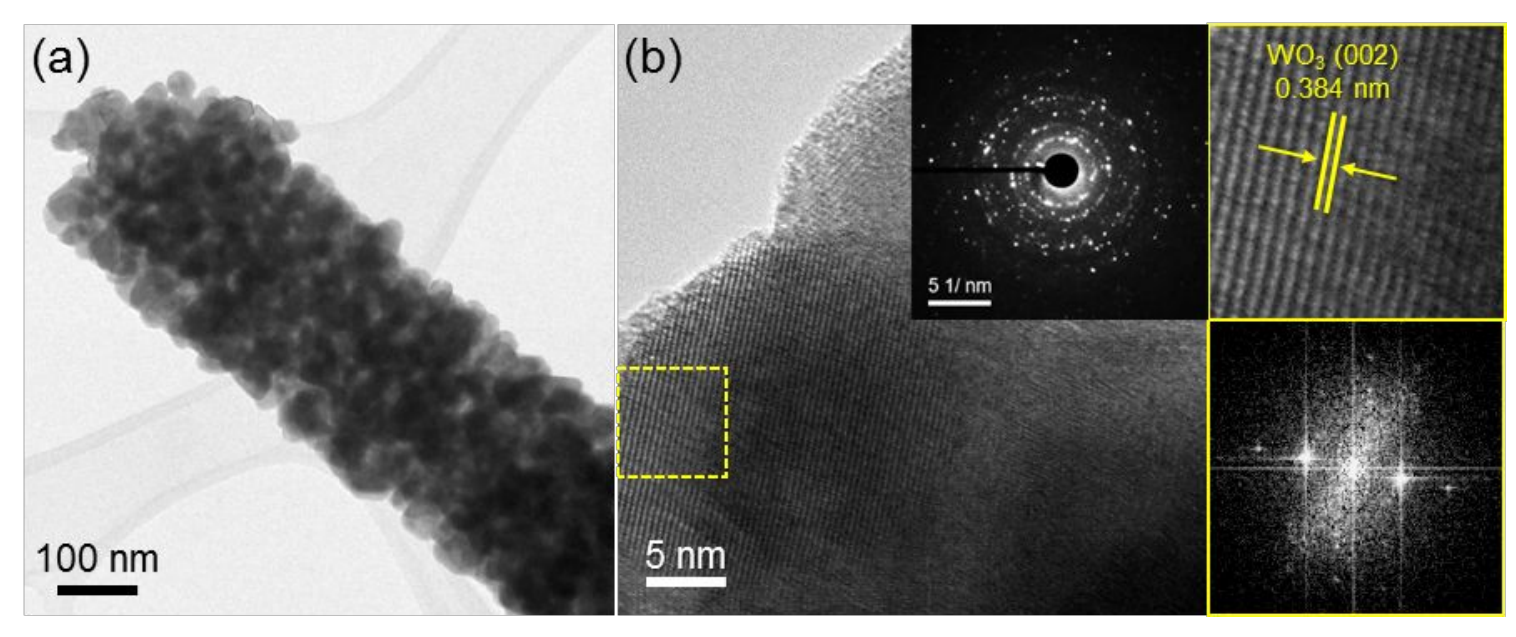

Figure S5. (a) TEM image of pristine $\mathrm{WO}_{3}$ NFs. (b) HRTEM image, magnified TEM image, and corresponding FFT pattern of pristine $\mathrm{WO}_{3}$ NFs. The SAED pattern is shown in the inset of (b). 

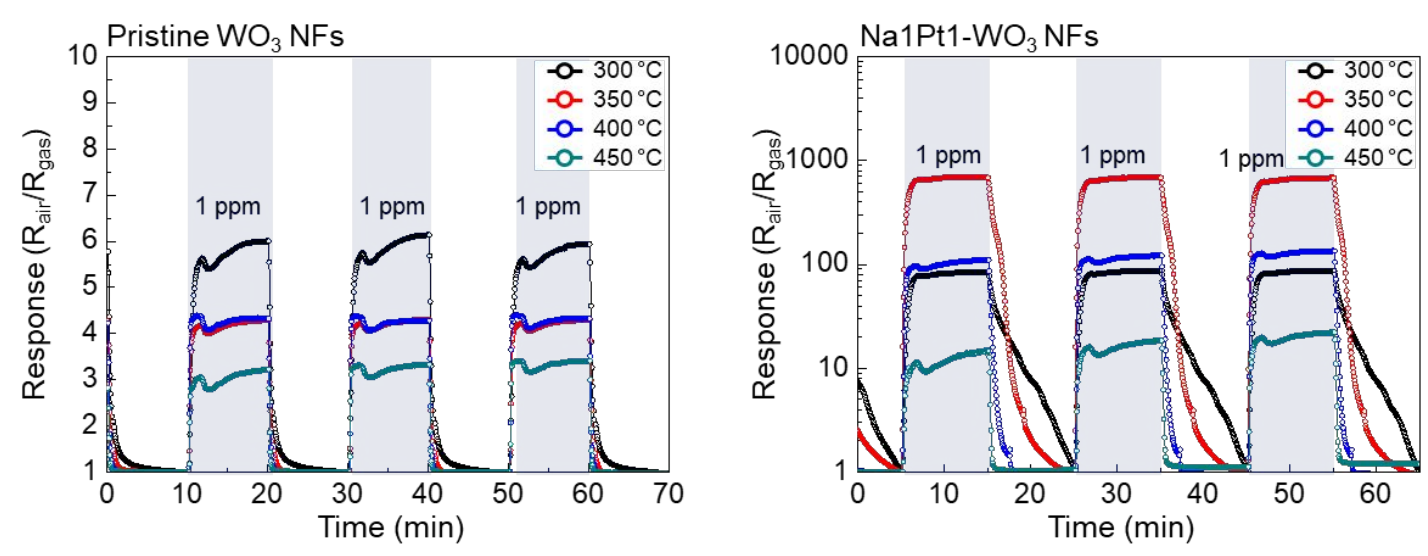

Figure S6. Sensing performance of (a) pristine $\mathrm{WO}_{3} \mathrm{NFs}$ and (b) $\mathrm{Na} 1 \mathrm{Pt} 1-\mathrm{WO}_{3} \mathrm{NFs}$ at 300 ,

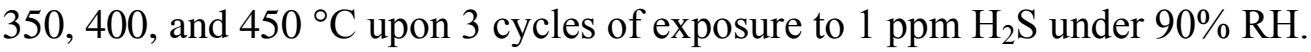




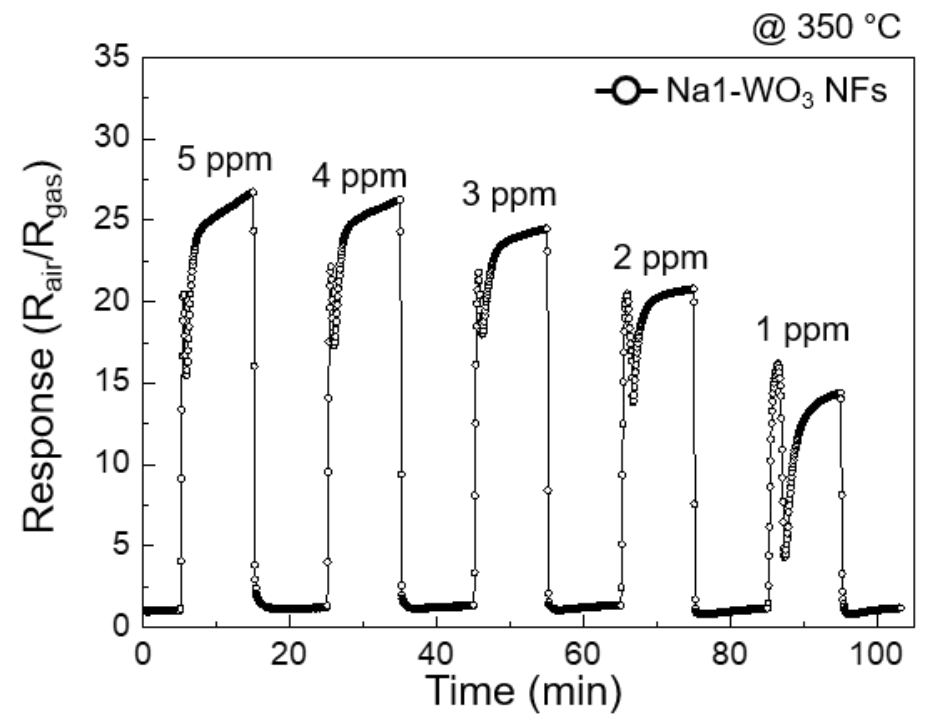

Figure S7. Sensing performance of $\mathrm{Na}^{-}-\mathrm{WO}_{3} \mathrm{NFs}$ at $350{ }^{\circ} \mathrm{C}$ toward 5-1 ppm $\mathrm{H}_{2} \mathrm{~S}$ under $90 \%$ RH. 

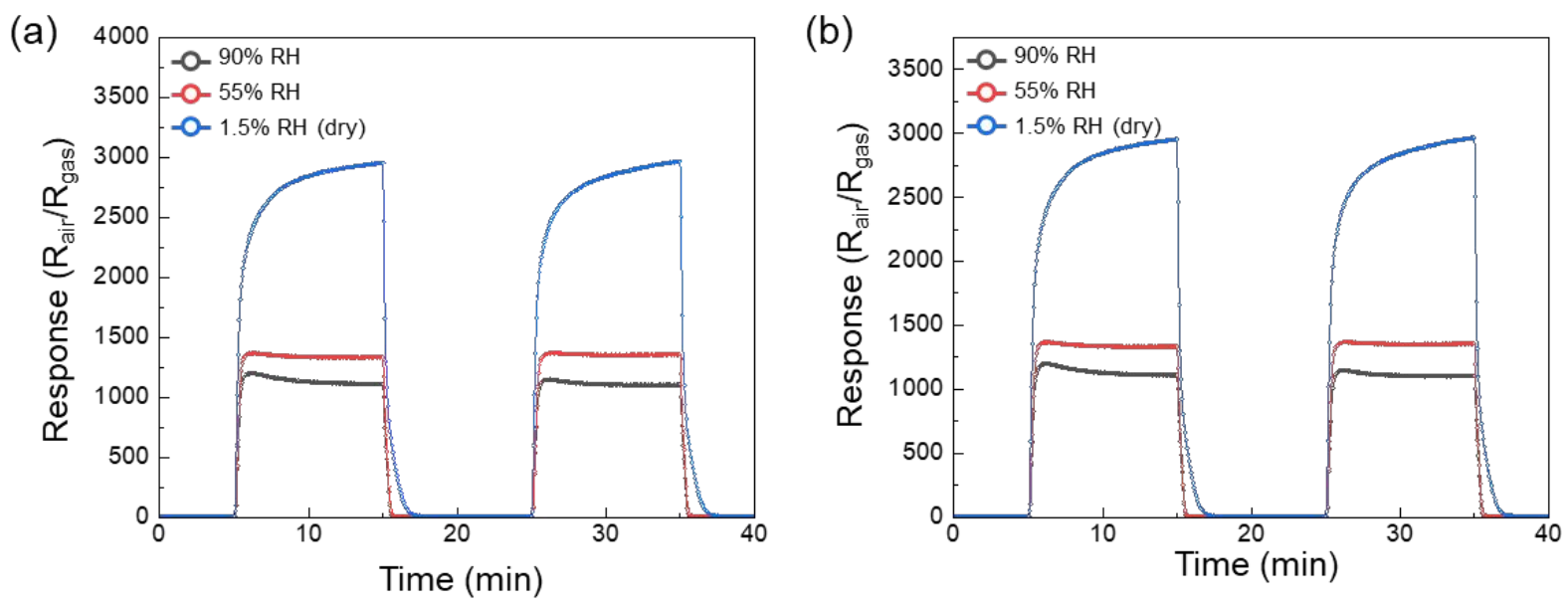

Figure S8. Comparison study of the response $\left(\mathrm{R}_{\text {air }} / \mathrm{R}_{\text {gas }}\right)$ values at $1.5 \%, 55 \%$ and $90 \%$ relative humidity levels for (a) Na1Pt2-WO $\mathrm{WF}_{3} \mathrm{NF}$, and (b) Na2Pt1- $\mathrm{WO}_{3} \mathrm{NFs}$.

For both samples, the response values are largely decreased when humidity is introduced. Water vapor competes with oxygen species upon adsorption onto the surface of the semiconducting metal oxides. As humidity level is increased, relative concentration of chemisorbed oxygen species on the metal oxides will decrease and therefore, the thickness of electron depletion layer formed on the surface of metal oxide nanograins is much smaller than that in dry air. ${ }^{1}$ 


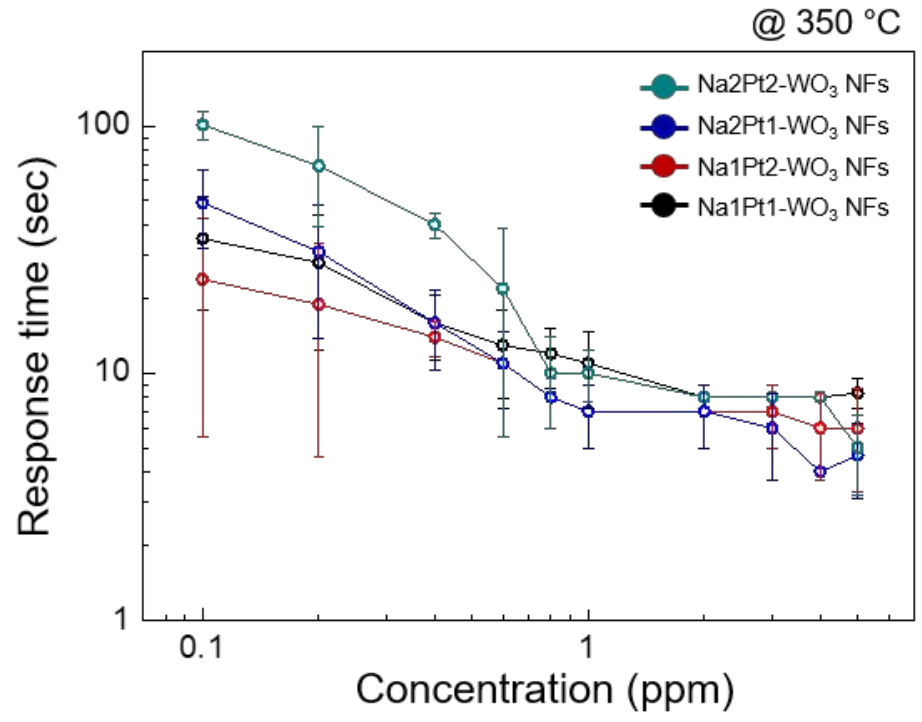

Figure S9. Response time versus $\mathrm{H}_{2} \mathrm{~S}$ concentration of $\mathrm{Na} 1 \mathrm{Pt} 1-\mathrm{WO}_{3} \mathrm{NFs}, \mathrm{Na} 1 \mathrm{Pt} 2-\mathrm{WO}_{3} \mathrm{NFs}$, $\mathrm{Na} 2 \mathrm{Pt} 1-\mathrm{WO}_{3} \mathrm{NFs}$, and $\mathrm{Na} 2 \mathrm{Pt} 2-\mathrm{WO}_{3} \mathrm{NFs}$ toward 5-0.1 ppm $\mathrm{H}_{2} \mathrm{~S}$ at $350{ }^{\circ} \mathrm{C}$ under $90 \% \mathrm{RH}$. 


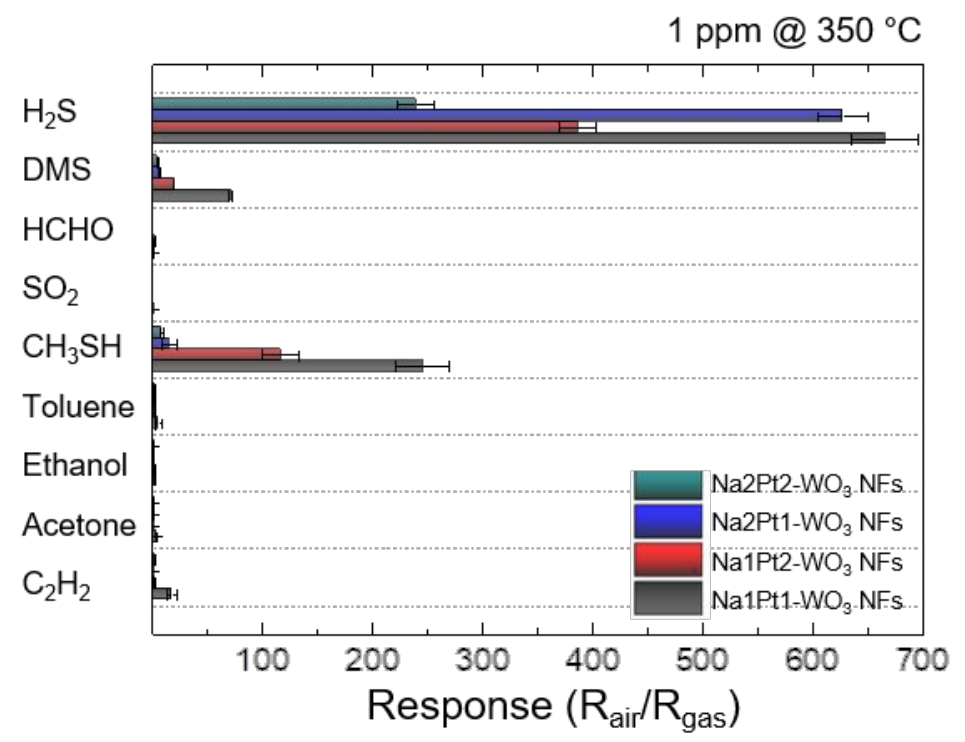

Figure S10. Selectivity of Na1Pt1-WO $\mathrm{W}_{3} \mathrm{NFs}, \mathrm{Na} 1 \mathrm{Pt} 2-\mathrm{WO}_{3} \mathrm{NFs}, \mathrm{Na} 2 \mathrm{Pt} 1-\mathrm{WO}_{3} \mathrm{NFs}$, and Na2$\mathrm{Pt} 2-\mathrm{WO}_{3} \mathrm{NFs}$ toward $1 \mathrm{ppm}$ of 9 different gas analytes at $350{ }^{\circ} \mathrm{C}$ under $90 \% \mathrm{RH}$. 

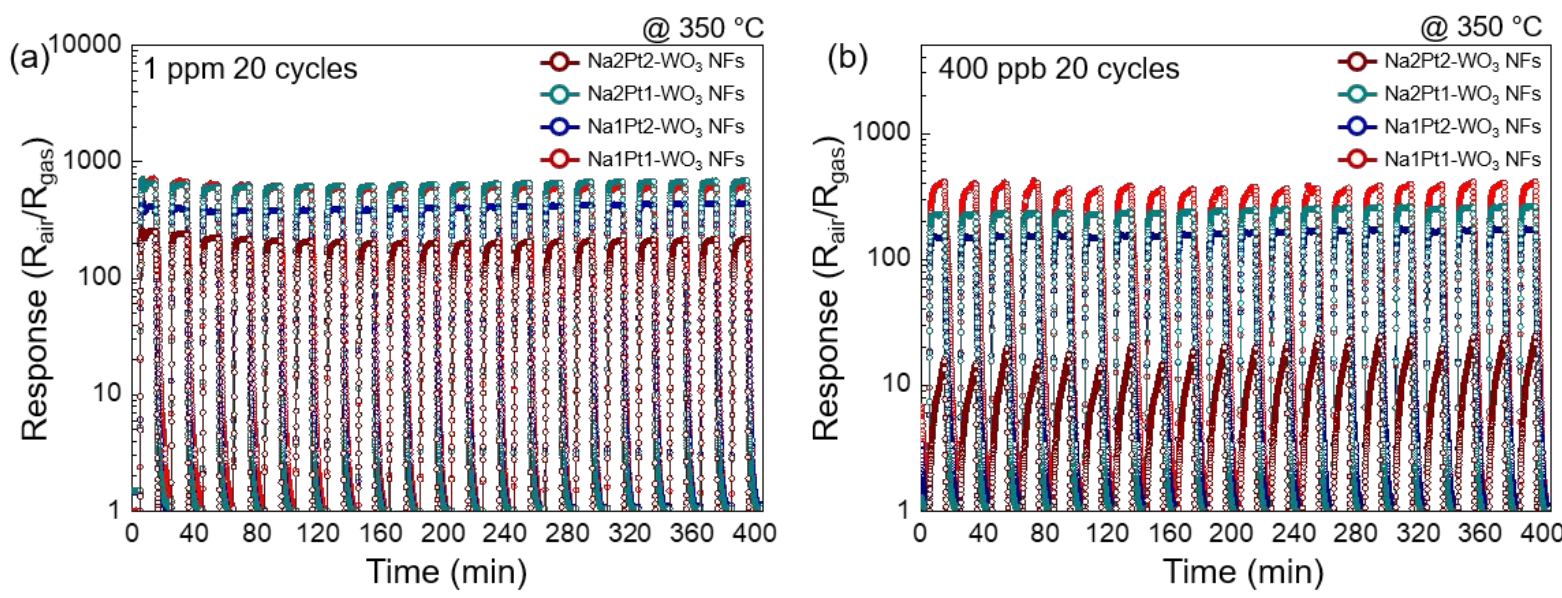

Figure S11. Long-term reliability tests of Na1Pt1- $\mathrm{WO}_{3} \mathrm{NFs}, \mathrm{Na} 1 \mathrm{Pt} 2-\mathrm{WO}_{3} \mathrm{NFs}, \mathrm{Na} 2 \mathrm{Pt} 1-\mathrm{WO}_{3}$ NFs, and Na2Pt2- $\mathrm{WO}_{3} \mathrm{NFs}$ toward 20 cycles of exposure to (a) $1 \mathrm{ppm} \mathrm{H}_{2} \mathrm{~S}$ and (b) $400 \mathrm{ppb}$ $\mathrm{H}_{2} \mathrm{~S}$ at $350{ }^{\circ} \mathrm{C}$ under $90 \% \mathrm{RH}$. 


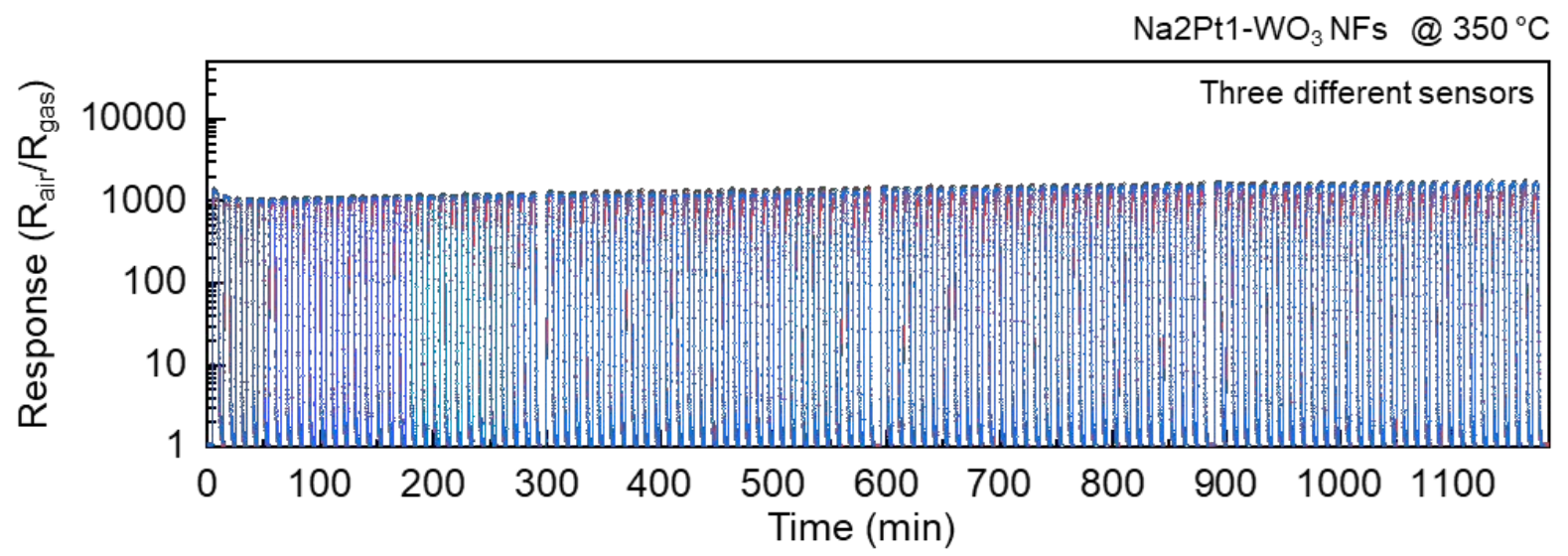

Figure S12. 116 cyclic exposure and recovery to $1 \mathrm{ppm} \mathrm{H}_{2} \mathrm{~S}$ at $350{ }^{\circ} \mathrm{C}$ for $\mathrm{Na} 2 \mathrm{Pt} 1-\mathrm{WO}_{3} \mathrm{NFs}$ sensors. 


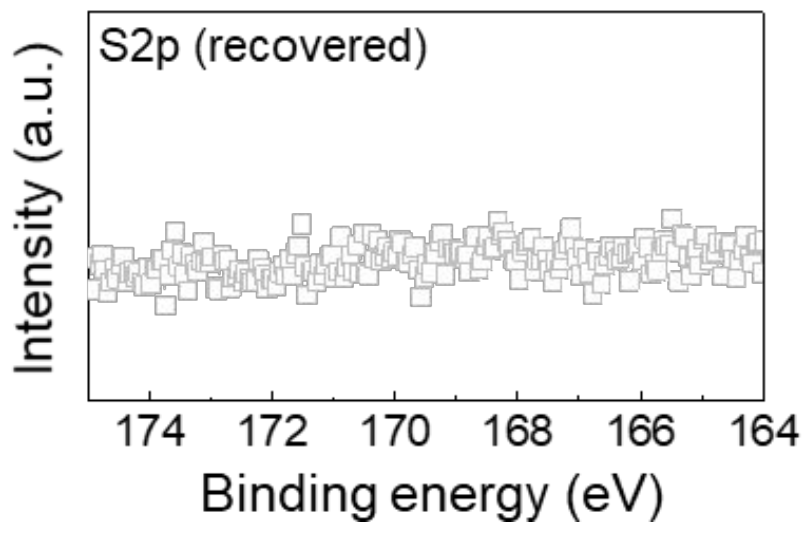

Figure S13. Ex-situ XPS analysis of $\mathrm{NaPt}-\mathrm{WO}_{3} \mathrm{NFs}$ after recovery from $\mathrm{H}_{2} \mathrm{~S}$ exposure in the vicinity of $\mathrm{S} 2 p$. 


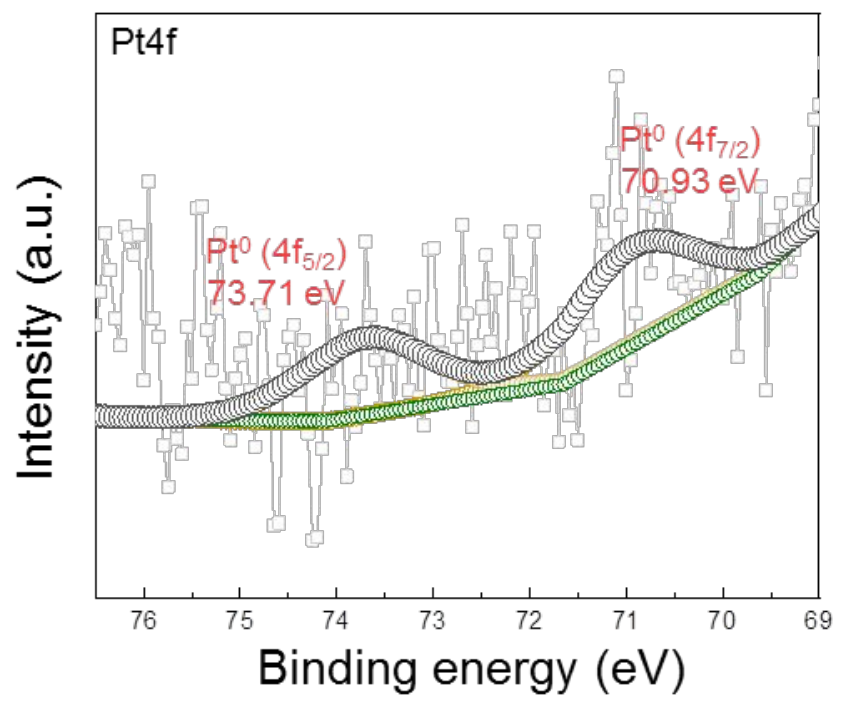

Figure S14. XPS analysis of Na1Pt1- $\mathrm{WO}_{3} \mathrm{NFs}$ in the vicinity of Pt $4 f$ peaks. 

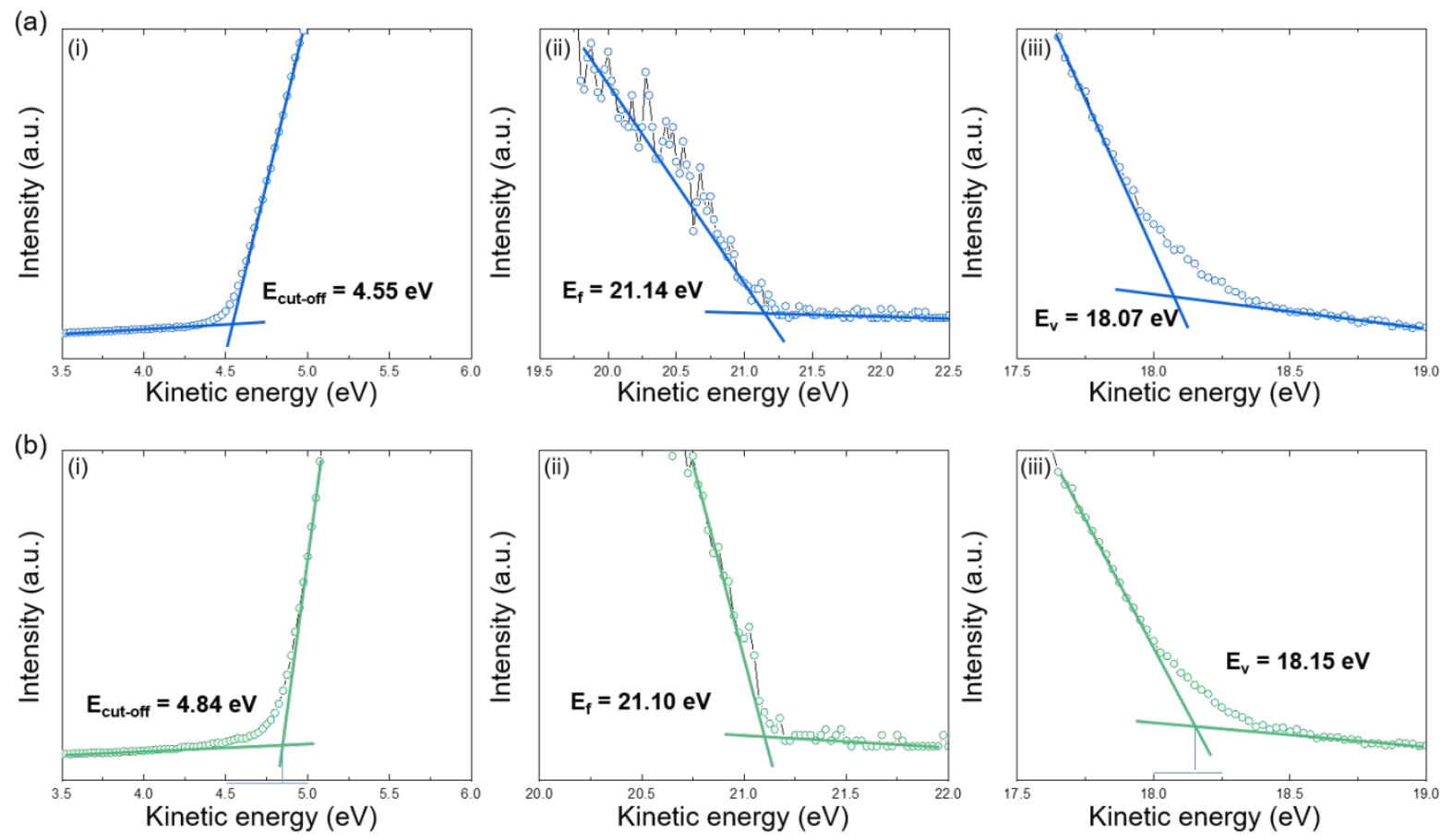

Figure S15. UPS analysis of (a) Na1Pt1- $\mathrm{WO}_{3} \mathrm{NFs}$ and (b) pristine $\mathrm{WO}_{3} \mathrm{NFs}$. For each sample: magnified spectra for the determination of (i) $E_{\text {cut-off, }}$ (ii) $E_{\mathrm{f}}$, and (iii) $E_{\mathrm{v}}$. 
(a)

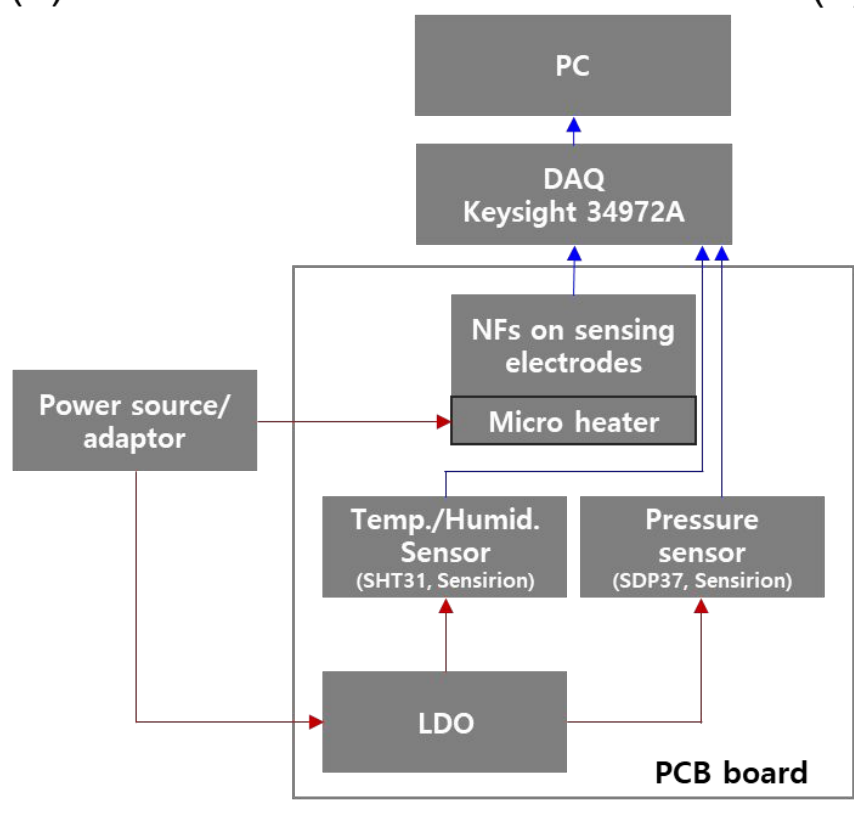

(b)

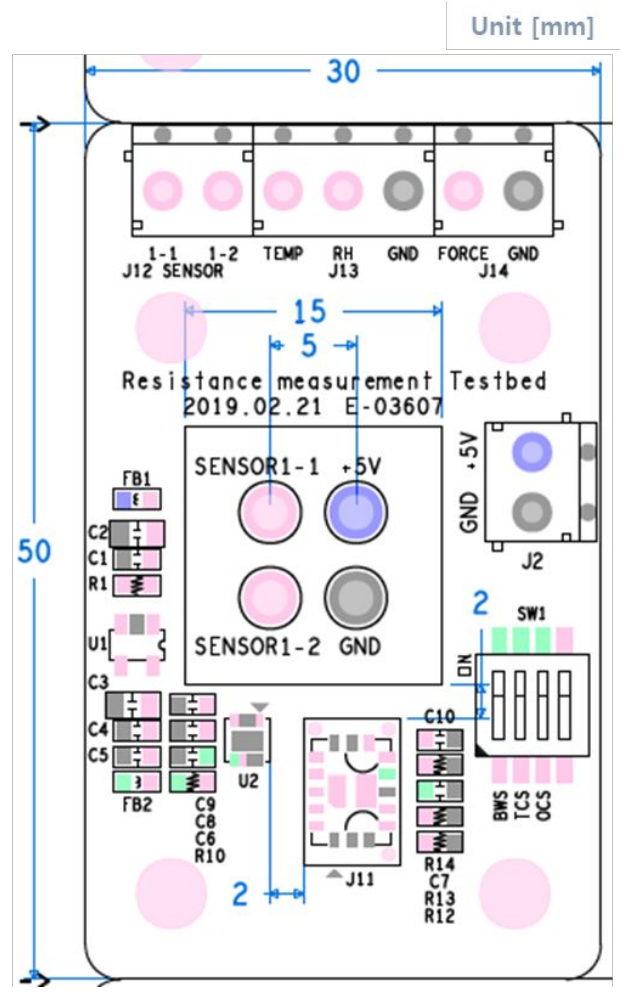

Figure S16. (a) Hardware block diagram of the overall system with the test device (gray box) and (b) PCB artwork of the test device. 


\section{Step 1}

- Insert a $1 \mathrm{~mL}$ syringe upto the stopper into the oral cavity and hold the syringe between the front teeth.

- Close mouth tightly. Wait 30 seconds to collect gas in oral cavity.

\section{Step 3}

- Inject the remaining oral gas into the inlet of the OralChroma.

- The measurement will start automatically.

\section{Step 2}

- Slowly pull the plunger. Push it in and pull it out at 3 times before removing the syringe from the mouth.

- Eject the remaining oral gas until it reaches $1 \mathrm{~mL}$ by pushing the plunge.

\section{Step 4}

- Read $\mathrm{H}_{2} \mathrm{~S}$ concentration in 4 minutes.

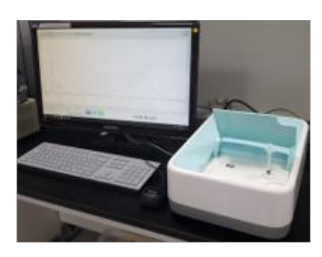

Figure S17. Detailed procedure for oral $\mathrm{H}_{2} \mathrm{~S}$ concentration measurement using the OralChroma system. 

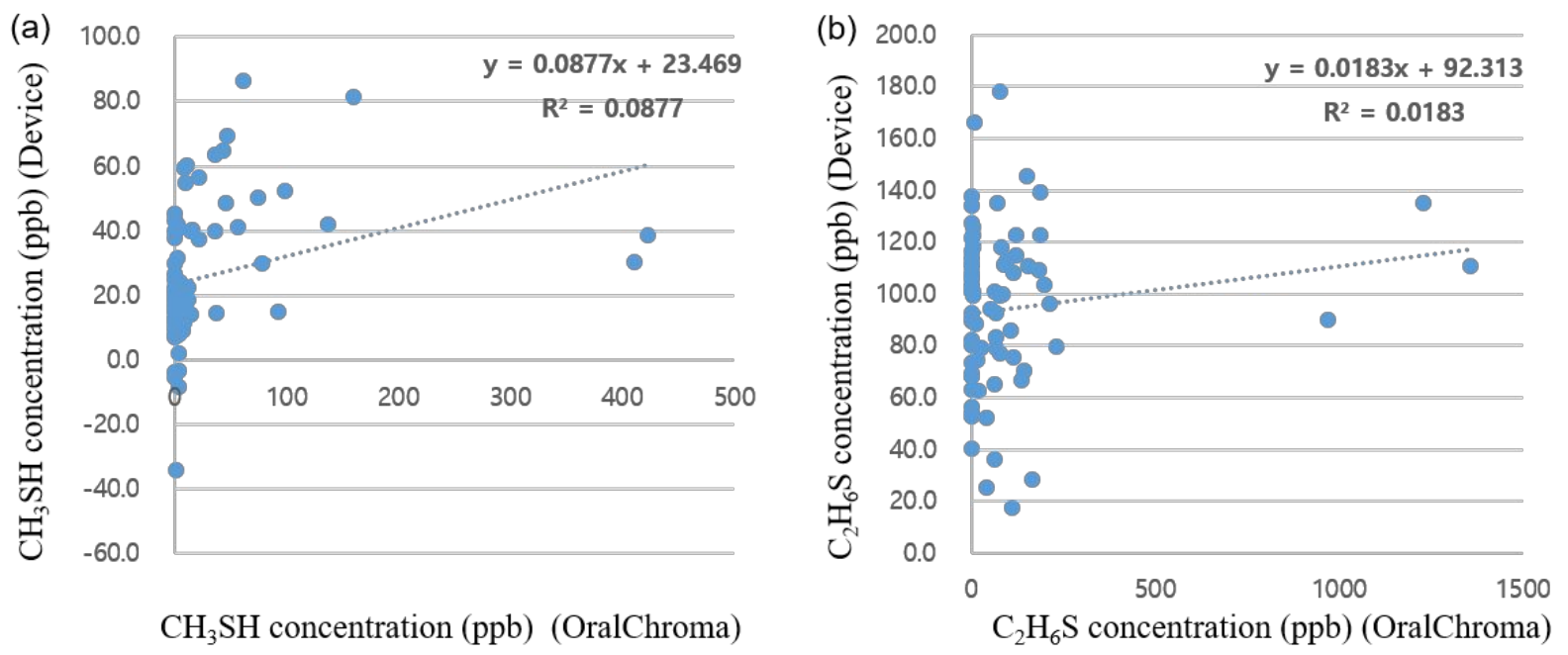

Figure S18. Correlation between $\mathrm{CH}_{3} \mathrm{SH}$ and $\mathrm{C}_{2} \mathrm{H}_{6} \mathrm{~S}$ concentration measured by the OralChroma (x-axis) and calculated by the fitting model (y-axis). 


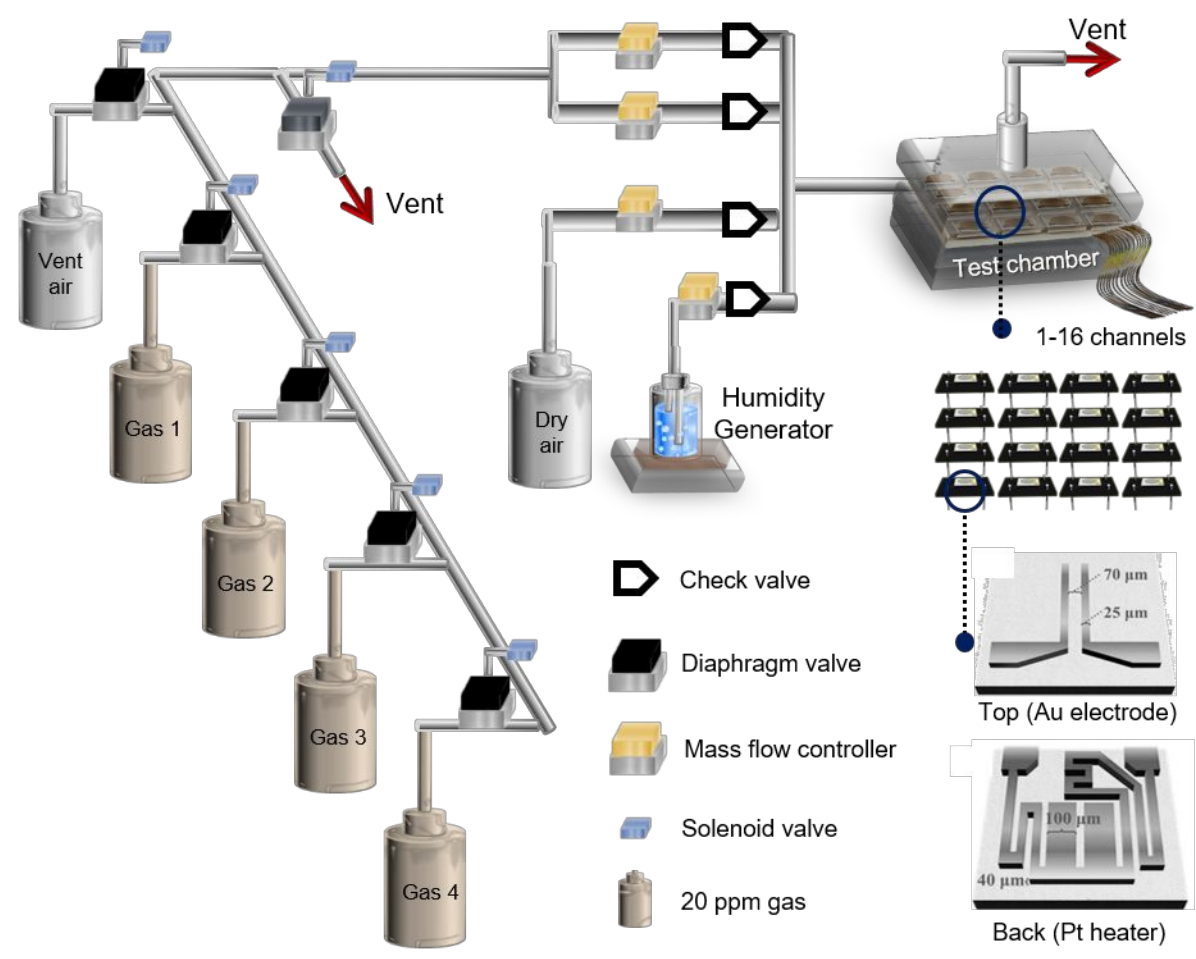

Figure S19. Schematic illustration of the gas sensor measurement setup. 
Table S1. Relative amounts (wt\%) of $\mathrm{Na}$ and $\mathrm{Pt}$ in the prepared samples with respect to $\mathrm{WO}_{3}$.

\begin{tabular}{ccc}
\hline Sample & Na Content $(\mathbf{w t} \%)$ & Pt Content $(\mathbf{w t} \%)$ \\
\hline Pristine $\mathrm{WO}_{3} \mathrm{NFs}$ & - & - \\
$\mathrm{Na} 1-\mathrm{WO}_{3} \mathrm{NFs}$ & 0.471 & - \\
$\mathrm{Pt1}-\mathrm{WO}_{3} \mathrm{NFs}$ & - & 0.013 \\
$\mathrm{Na} 1 \mathrm{Pt} 1-\mathrm{WO}_{3} \mathrm{NFs}$ & 0.471 & 0.013 \\
$\mathrm{Na} 1 \mathrm{Pt} 2-\mathrm{WO}_{3} \mathrm{NFs}$ & 0.471 & 0.026 \\
$\mathrm{Na} 2 \mathrm{Pt} 1-\mathrm{WO}_{3} \mathrm{NFs}$ & 0.942 & 0.013 \\
$\mathrm{Na} 2 \mathrm{Pt} 2-\mathrm{WO}_{3} \mathrm{NFs}$ & 0.942 & 0.026 \\
\hline
\end{tabular}


Table S2. Raw $\mathrm{H}_{2} \mathrm{~S}, \mathrm{CH}_{3} \mathrm{SH}$, and $\mathrm{C}_{2} \mathrm{H}_{6} \mathrm{~S}$ concentration data obtained by direct, real-time human breath experiments on 80 cases from 8 subjects using OralChroma and the test device.

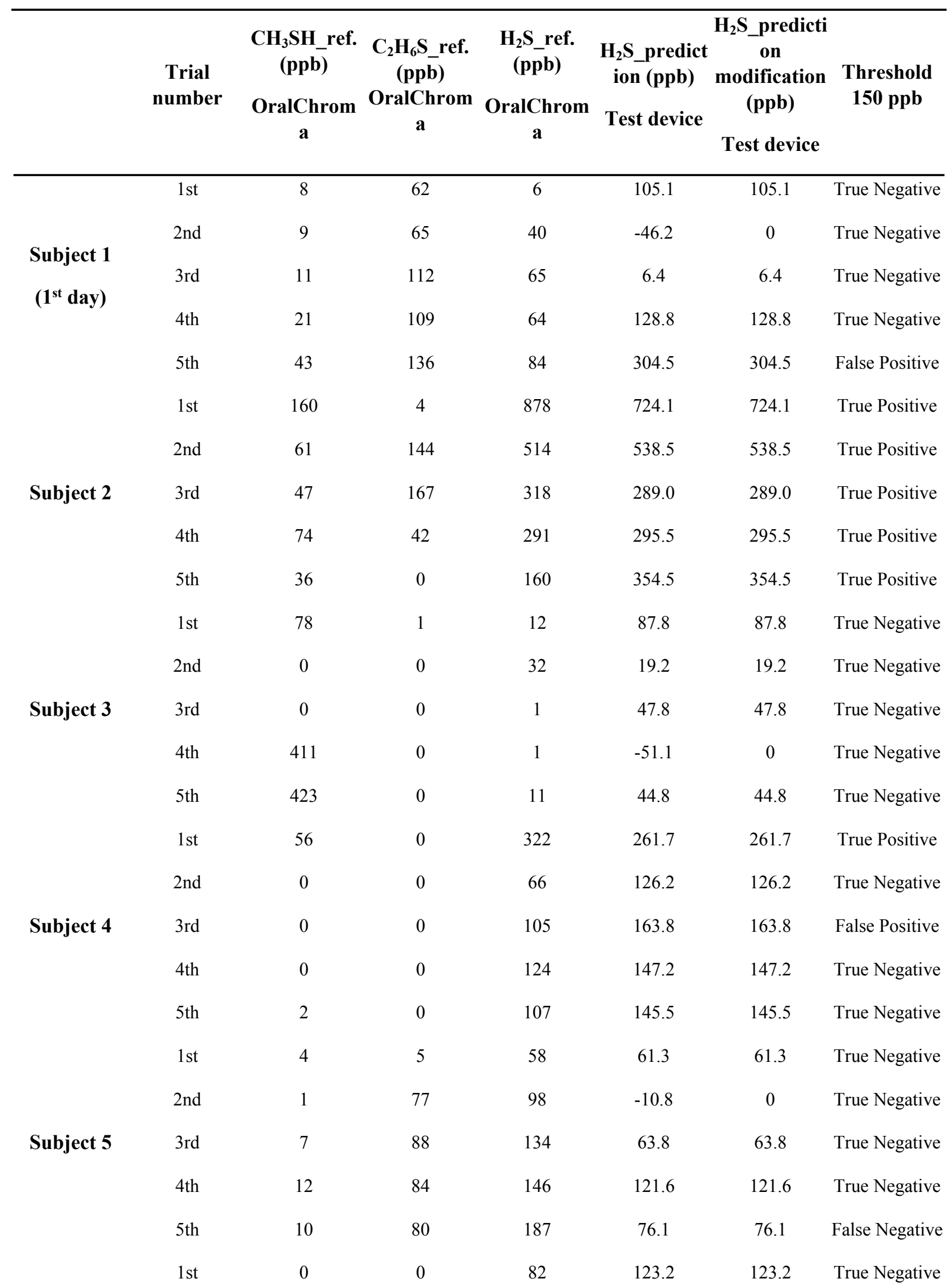




\begin{tabular}{|c|c|c|c|c|c|c|c|}
\hline \multirow[t]{6}{*}{ Subject 6} & 2nd & 0 & 1228 & 22 & 93.1 & 93.1 & True Negative \\
\hline & $3 \mathrm{rd}$ & 0 & 1358 & 21 & 92.6 & 92.6 & True Negative \\
\hline & 4 th & 0 & 0 & 22 & 83.9 & 83.9 & True Negative \\
\hline & 5 th & 0 & 970 & 32 & 155.1 & 155.1 & False Positive \\
\hline & $1 \mathrm{st}$ & 0 & 6 & 9 & 54.2 & 54.2 & True Negative \\
\hline & $2 \mathrm{nd}$ & 0 & 0 & 10 & 49.0 & 49.0 & True Negative \\
\hline \multirow[t]{5}{*}{ Subject 7} & $3 \mathrm{rd}$ & 0 & 200 & 53 & 68.4 & 68.4 & True Negative \\
\hline & 4 th & 0 & 122 & 10 & 99.5 & 99.5 & True Negative \\
\hline & 5 th & 0 & 187 & 44 & 39.7 & 39.7 & True Negative \\
\hline & $1 \mathrm{st}$ & 0 & 81 & 16 & 171.6 & 171.6 & False Positive \\
\hline & $2 \mathrm{nd}$ & 4 & 0 & 11 & -2.1 & 0 & True Negative \\
\hline \multirow[t]{4}{*}{ Subject 8} & $3 \mathrm{rd}$ & 8 & 28 & 17 & -26.4 & 0 & True Negative \\
\hline & 4 th & 3 & 43 & 9 & -9.9 & 0 & True Negative \\
\hline & 5 th & 0 & 67 & 4 & 33.3 & 33.3 & True Negative \\
\hline & $1 \mathrm{st} \_1$ & 2 & 0 & 2 & 19.2 & 19.2 & True Negative \\
\hline \multirow{6}{*}{$\begin{array}{l}\text { Subject } 1 \\
\left(2^{\text {nd }} \text { day) }\right.\end{array}$} & 2nd_1 & 0 & 114 & 8 & -23.6 & 0 & True Negative \\
\hline & 3rd_1 & 4 & 0 & 10 & 95.4 & 95.4 & True Negative \\
\hline & 4th_1 & 15 & 0 & 14 & 12.4 & 12.4 & True Negative \\
\hline & 5th_1 & 14 & 0 & 52 & -3.9 & 0 & True Negative \\
\hline & 1st_1 & 137 & 7 & 651 & 371.5 & 371.5 & True Positive \\
\hline & 2nd_1 & 21 & 0 & 204 & 269.6 & 269.6 & True Positive \\
\hline \multirow[t]{5}{*}{ Subject 2} & 3rd_1 & 45 & 0 & 195 & 211.9 & 211.9 & True Positive \\
\hline & 4th_1 & 98 & 7 & 498 & 321.3 & 321.3 & True Positive \\
\hline & 5th_1 & 36 & 69 & 352 & 188.1 & 188.1 & True Positive \\
\hline & 1st_1 & 3 & 70 & 66 & 47.3 & 47.3 & True Negative \\
\hline & 2nd_1 & 0 & 0 & 60 & 58.3 & 58.3 & True Negative \\
\hline \multirow[t]{3}{*}{ Subject 3} & 3rd_1 & 0 & 153 & 76 & 86.6 & 86.6 & True Negative \\
\hline & 4th_1 & 10 & 189 & 157 & 65.5 & 65.5 & False Negative \\
\hline & 5th_1 & 12 & 0 & 120 & 32.9 & 32.9 & True Negative \\
\hline \multirow{2}{*}{ Subject 4} & 1st_1 & 1 & 0 & 40 & 153.2 & 153.2 & False Positive \\
\hline & 2nd 1 & 0 & 0 & 45 & 104.0 & 104.0 & True Negative \\
\hline
\end{tabular}




\begin{tabular}{|c|c|c|c|c|c|c|c|}
\hline & 3rd_1 & 5 & 63 & 157 & 132.5 & 132.5 & False Negative \\
\hline & 4th_1 & 5 & 96 & 176 & 91.0 & 91.0 & False Negative \\
\hline & 5th_1 & 0 & 154 & 72 & 19.0 & 19.0 & True Negative \\
\hline & 1st_1 & 0 & 7 & 82 & 216.1 & 216.1 & False Positive \\
\hline & 2nd_1 & 9 & 14 & 139 & 137.0 & 137.0 & True Negative \\
\hline \multirow[t]{5}{*}{ Subject 5} & 3rd_1 & 14 & 17 & 185 & 155.6 & 155.6 & True Positive \\
\hline & 4th_1 & 37 & 20 & 293 & 110.6 & 110.6 & False Negative \\
\hline & 5th_1 & 0 & 3 & 73 & 129.3 & 129.3 & True Negative \\
\hline & 1st_1 & 3 & 8 & 8 & 57.9 & 57.9 & True Negative \\
\hline & 2nd_1 & 0 & 186 & 13 & 63.6 & 63.6 & True Negative \\
\hline \multirow[t]{5}{*}{ Subject 6} & 3rd_1 & 0 & 121 & 15 & 110.2 & 110.2 & True Negative \\
\hline & 4th_1 & 2 & 0 & 33 & 1.9 & 1.9 & True Negative \\
\hline & 5th_1 & 1 & 0 & 32 & 9.8 & 9.8 & True Negative \\
\hline & 1st_1 & 0 & 2 & 7 & 138.4 & 138.4 & True Negative \\
\hline & 2nd_1 & 0 & 214 & 4 & 61.0 & 61.0 & True Negative \\
\hline \multirow[t]{5}{*}{ Subject 7} & 3rd_1 & 0 & 232 & 21 & 53.1 & 53.1 & True Negative \\
\hline & 4th_1 & 0 & 78 & 109 & 96.1 & 96.1 & True Negative \\
\hline & 5th_1 & 0 & 0 & 98 & 57.0 & 57.0 & True Negative \\
\hline & 1st_1 & 0 & 51 & 4 & -75.2 & 0 & True Negative \\
\hline & 2nd_1 & 0 & 69 & 3 & 28.3 & 28.3 & True Negative \\
\hline \multirow[t]{3}{*}{ Subject 8} & 3rd_1 & 0 & 113 & 7 & 14.3 & 14.3 & True Negative \\
\hline & 4th_1 & 92 & 0 & 3 & 14.2 & 14.2 & True Negative \\
\hline & 5th_1 & 3 & 0 & 10 & 7.3 & 7.3 & True Negative \\
\hline
\end{tabular}


Table S3. Accuracy of the test device compared with the OralChroma results.

\begin{tabular}{|c|c|c|c|c|}
\hline & \multicolumn{3}{|l|}{ OralChroma } \\
\hline & & Positive & Negative & Sum \\
\hline \multirow{4}{*}{$\begin{array}{l}\text { Test } \\
\text { device }\end{array}$} & Positive & $\begin{array}{l}12 \\
\text { (True Positive, TP) }\end{array}$ & $\begin{array}{l}6 \\
\text { (False Positive, FP) }\end{array}$ & 18 \\
\hline & Negative & $\begin{array}{l}5 \\
\text { (False Negative, FN) }\end{array}$ & $\begin{array}{l}57 \\
\text { (True Negative, TN) }\end{array}$ & 62 \\
\hline & Sum & 17 & 63 & 80 \\
\hline & & $\begin{array}{l}\text { Sensitivity }(\%)= \\
\mathrm{TP} /(\mathrm{TP}+\mathrm{FN})=70.6\end{array}$ & $\begin{array}{l}\text { Specificity }(\%)= \\
\mathrm{TN} /(\mathrm{FP}+\mathrm{TN})=90.5\end{array}$ & $\begin{array}{l}\text { Accuracy }(\%)= \\
(\mathrm{TP}+\mathrm{TN}) / \text { Sum }= \\
86.3\end{array}$ \\
\hline
\end{tabular}




\section{Reference}

1. Bârsan N.; Weimar, U. Understanding the Fundamental Principles of Metal Oxide Based Gas Sensors; The Example of CO Sensing with $\mathrm{SnO}_{2}$ Sensors in the Presence of Humidity. J. Phys. Condens. Matter 2003, 15, 813-839 